\title{
Stability of SIRS Epidemic Model with Stochastic Perturbation and Distributed Delays
}

\author{
Ramziya Rifhat, Xamxinur Abdurahman, and Ahmadjan Muhammadhaji
}

College of Mathematics and System Sciences, Xinjiang University, Urumqi 830046, China

Correspondence should be addressed to Xamxinur Abdurahman; xamxinur@sina.com.cn

Received 18 April 2013; Accepted 26 May 2013

Academic Editors: K. Djidjeli, J. R. Fernandez, and F. Jauberteau

Copyright (C) 2013 Ramziya Rifhat et al. This is an open access article distributed under the Creative Commons Attribution License, which permits unrestricted use, distribution, and reproduction in any medium, provided the original work is properly cited.

A class of SIRS epidemic model with stochastic perturbation and distributed delays is proposed and discussed. Some sufficient conditions on the stability of the zero solution are established. Finally, concluding that, the white noise is favorable for the stability of zero solution and the distributed time delays have no impact on the stability of zero solution.

\section{Introduction}

As an important class of mathematical models, stochastic differential equations have been widely used in automatic control, biological, chemical reaction engineering, medicine, economics, demography, and many other scientific fields. For better application, people have done a lot of work in the stochastic differential equations and various particular equations proposed for the study of stochastic differential equations. Subsequently, a lot of the literature related to this topic was published and extensive research results were obtained. However, many of the problems have not been fully investigated and deserve further study. As it is well known, in recent years, the stochastic epidemic systems are extensively studied. Many important and influential results have been established and can be found in many articles and books. Particularly, the stability of zero solutions for various type stochastic epidemic systems has been extensively studied in [1-7] and the references are cited therein.

In [8], the authors studied the following classical SIRS epidemiological model with distributed time delay:

$$
\begin{gathered}
\dot{S}(t)=-\beta S(t) \int_{0}^{h} f(s) I(t-s) \mathrm{d} s-\mu_{1} S(t)+b+k R(t), \\
\dot{I}(t)=\beta S(t) \int_{0}^{h} f(s) I(t-s) \mathrm{d} s-\left(\mu_{2}+\lambda\right) I(t), \\
\dot{R}(t)=\lambda I(t)-\left(\mu_{3}+k\right) R(t) .
\end{gathered}
$$

Individual are susceptible, then infectious, recovered with temporary immunity, and then susceptible again when the immunity is lost. Then parameter $b>0$ is the rate of susceptible individuals recruited into the population (either by birth or immigration) and $\mu_{1}>0$ is the natural death rate. The parameter $\lambda>0$ describes the rate at which the infectious population becomes recovered, and $k>0$ denotes the rate at which the recovered population loses immunity. The positive constant $\beta>0$ is the average number of contacts per infective per day. The nonnegative constant $h$ is the time delay. $f(s)$ is the fraction of vector population for which the time taken to become infectious, and it is assumed to be a nonnegative function on $[0, h]$ as density function and satisfies $\int_{0}^{h} f(s) \mathrm{d} s=$ 1. For detailed biological background, we refer to $[8,9]$.

In [8], the authors derived the disease-free equilibrium $P^{0}\left(S^{0}, 0,0\right)$ and the basic reproductive number $R_{0}=$ $\left(\beta b / \mu_{1}\left(\lambda+\mu_{2}\right)\right)$ of system $(1)$, where $S^{0}=\left(b / \mu_{1}\right), \beta$ is the transmission coefficient, and $1 /\left(\lambda+\mu_{2}\right)$ is the average residence time in the infectious individuals class. By straightforward computations, it can be seen that, for $R_{0}>1$, system (1) has a unique endemic equilibrium state $P^{*}\left(S^{*}, I^{*}, R^{*}\right)$ in the interior of $\mathfrak{R}_{+}^{3}$, where

$$
\begin{gathered}
S^{*}=\frac{S^{0}}{R_{0}}=\frac{\lambda+\mu_{2}}{\beta}, \\
I^{*}=\frac{k+\mu_{3}}{\mu_{1}\left(k+\lambda+\mu_{2}\right)}\left(b-\mu_{1} S^{*}\right),
\end{gathered}
$$




$$
R^{*}=\lambda \frac{I^{*}}{k+\mu_{3}}
$$

On the other hand, white noise stochastic perturbations around the positive endemic equilibrium of epidemic models were considered in $[1,3]$. In [1], the authors studied the following stochastic differential equation model:

$$
\begin{gathered}
\dot{S}(t)=-\beta S(t) \int_{0}^{h} f(s) I(t-s) \mathrm{d} s-\mu_{1} S(t) \\
\quad+b+\sigma_{1}\left(S(t)-S^{*}\right) \dot{w}_{1}(t), \\
\dot{I}(t)=\beta S(t) \int_{0}^{h} f(s) I(t-s) \mathrm{d} s-\left(\mu_{2}+\lambda\right) I(t) \\
+\sigma_{2}\left(I(t)-I^{*}\right) \dot{w}_{2}(t), \\
\dot{R}(t)=\lambda I(t)-\mu_{3} R(t)+\sigma_{3}\left(R(t)-R^{*}\right) \dot{w}_{3}(t),
\end{gathered}
$$

where $S^{*}, I^{*}$, and $R^{*}$ are the positive points of equilibrium for the corresponding deterministic system (1), constants $\sigma_{i}(i=1,2)$ are the intensity of white noise, and $w_{i}(t)(i=$ $1,2,3)$ are the standard Wiener processes. In [1], the authors used the Lyapunov functionals method and the sufficient conditions for the stability of the zero solution are obtained for system (3).

Likewise, in [3], the authors studied the same type of stochastic perturbations and extended the deterministic model for the epidemics induced by virulent phages on bacteria in marine environment, allowing random fluctuations around the positive equilibrium, and concluded that the solution of the corresponding stochastic model for phage bacteria interaction was asymptotically mean square stable both analytically and numerically.

Motivated by the aforementioned works, in this paper,we consider the following SIRS epidemic model with stochastic perturbation and distributed delays:

$$
\begin{gathered}
\dot{S}(t)=-\beta S(t) \int_{0}^{h} f(s) I(t-s) \mathrm{d} s-\mu_{1} S(t)+b+k R(t) \\
+\sigma_{1}\left(S(t)-S^{*}\right) \dot{w}_{1}(t), \\
\dot{I}(t)=\beta S(t) \int_{0}^{h} f(s) I(t-s) \mathrm{d} s-\left(\mu_{2}+\lambda\right) I(t) \\
\quad+\sigma_{2}\left(I(t)-I^{*}\right) \dot{w}_{2}(t), \\
\dot{R}(t)=\lambda I(t)-\left(\mu_{3}+k\right) R(t)+\sigma_{3}\left(R(t)-R^{*}\right) \dot{w}_{3}(t),
\end{gathered}
$$

where the constants $\sigma_{1}, \sigma_{2}$, and $\sigma_{3}$ are the intensity of white noise $\dot{w}_{1}(t), \dot{w}_{2}(t), \dot{w}_{3}(t)$ and $w_{1}(t), w_{2}(t), w_{3}(t)$ are standard Wiener process defined on a complete probability space $\left(\Omega, \mathscr{F},\left\{\mathscr{F}_{t}\right\}_{t \geq 0}, P\right)$. The detailed definition of Wiener process can be found in [2].
Let $u_{1}=S-S^{*}, u_{2}=I-I^{*}, u_{3}=R-R^{*}$, where $N^{*}=$ $S^{*}+I^{*}+R^{*}$. Then, system (4) is rewritten in the following form:

$$
\begin{aligned}
\dot{u}_{1}= & -\left(\beta I^{*}+\mu_{1}\right) u_{1}-\beta S^{*} \int_{0}^{h} f(s) u_{2}(t-s) \mathrm{d} s-\beta u_{1} \\
& \times \int_{0}^{h} f(s) u_{2}(t-s) \mathrm{d} s+k u_{3}+\sigma_{1} u_{1} \dot{w}_{1}, \\
\dot{u}_{2}= & \beta I^{*} u_{1}-\beta S^{*} u_{2}+\beta S^{*} \int_{0}^{h} f(s) u_{2}(t-s) \mathrm{d} s+\beta u_{1} \\
& \times \int_{0}^{h} f(s) u_{2}(t-s) \mathrm{d} s+\sigma_{2} u_{2} \dot{w}_{2}, \\
& \dot{u}_{3}=\lambda u_{2}-\left(\mu_{3}+k\right) u_{3}+\sigma_{3} u_{3} \dot{w}_{3} .
\end{aligned}
$$

It is easy to see that the stability of zero solution in system (4) is equivalent to the stability of zero solution in system (5). In order to obtain the sufficient conditions for the stability of zero solution in system (5), we will consider the following linear part of system (5):

$$
\begin{gathered}
\dot{z}_{1}=-\left(\beta I^{*}+\mu_{1}\right) z_{1}-\beta S^{*} \int_{0}^{h} f(s) z_{2}(t-s) \mathrm{d} s \\
+k z_{3}+\sigma_{1} z_{1} \dot{w}_{1} \\
\dot{z}_{2}=\beta I^{*} z_{1}-\beta S^{*} z_{2}+\beta S^{*} \int_{0}^{h} f(s) z_{2}(t-s) \mathrm{d} s+\sigma_{2} z_{2} \dot{w}_{2}, \\
\dot{z}_{3}=\lambda z_{2}-\left(\mu_{3}+k\right) z_{3}+\sigma_{3} z_{3} \dot{w}_{3},
\end{gathered}
$$

and the auxiliary system without delays

$$
\begin{gathered}
\dot{y}_{1}=-\left(\beta I^{*}+\mu_{1}\right) y_{1}+k y_{3}+\sigma_{1} y_{1} \dot{w}_{1}, \\
\dot{y}_{2}=\beta I^{*} y_{1}-\beta S^{*} y_{2}+\sigma_{2} y_{2} \dot{w}_{2}, \\
\dot{y}_{3}=\lambda y_{2}-\left(\mu_{3}+k\right) y_{3}+\sigma_{3} y_{3} \dot{w}_{3} .
\end{gathered}
$$

\section{Preliminaries}

Consider the following stochastic differential equation [2]:

$$
\mathrm{d} x(t)=a\left(t, x_{t}\right) \mathrm{d} t+b\left(t, x_{t}\right) \mathrm{d} w(t), \quad x_{0}=\varphi \in H,
$$

where function $a\left(t, x_{t}\right)$ defined in $\left[t_{0}, \infty\right) \times R^{n}$ and $b\left(t, x_{t}\right)$ is an $n \times m$ matrix, $x_{t}(s)=x(t+s)$ with $s \leq 0, w(t)$ is $m$ dimensional Wiener process. We assume that $x=0$ is a trivial solution of system (8), that is, $a(t, 0)=0, b(t, 0)=0$ for all $t \geq$ $t_{0}$. Denote with $H$ the space of $f_{0}$-adapted random variables $\varphi$, with $\varphi(s) \in \mathfrak{R}^{n}, s \leq 0$ and $\|\varphi\|_{0}=\sup _{s \leq 0}|\varphi(s)|,\|\varphi\|_{1}^{2}=$ $\sup _{s \leq 0} \mathbb{E}|\varphi(s)|^{2}$ (E is the mathematical expectation). Let $V$ : $[0, \infty) \times H \rightarrow R$ be a functional defined for $t \geq 0, \varphi \in H$.

Generating operator $L$ of (8) is defined [2] by formula

$$
L V(t, \varphi)=\lim _{\Delta \rightarrow 0} \frac{\mathbb{E}_{t, \varphi} V\left(t+\Delta, y_{t+\Delta}\right)-V(t, \varphi)}{\Delta},
$$


where a scalar functional $V(t, \varphi)$ is defined by $t \geq 0, \varphi \in H$, and $x(s)$ is the solution of (8) by $s \geq t$ with initial function $x_{t}=\varphi \in H$. Let us describe one class of functionals $V(t, \varphi)$ for which the operator $L$ can be calculated in final form. We reduce the arbitrary functional $V(t, \varphi), t \geq 0, \varphi \in H$, to the form $V(t, \varphi)=V(t, \varphi(0), \varphi(s)), s<0$ and define the function

$$
\begin{gathered}
V_{\varphi}(t, x)=V(t, \varphi)=V(t, x, x(t+s)), \\
s<0, \quad \varphi=x_{t}, \quad x=\varphi(0)=x(t) .
\end{gathered}
$$

Let $D$ be the class of functionals $V(t, \varphi)$ for which functional $V_{\varphi}(t, x)$ has two continuous derivations with respect to $x$ and one bounded derivative with respect to $t$ for almost all $t \geq 0$. For functionals from $D$ the generating operator $L$ of (8) is defined and is equal to the following:

$$
\begin{aligned}
L V\left(t, x_{t}\right)= & \frac{\partial V_{\varphi}(t, x)}{\partial t}+a^{\prime}\left(t, x_{t}\right) \frac{\partial V_{\varphi}(t, x)}{\partial x} \\
& +\frac{1}{2} \operatorname{Tr}\left[b^{\prime}\left(t, x_{t}\right) \frac{\partial^{2} V_{\varphi}(t, x)}{\partial x^{2}} b\left(t, x_{t}\right)\right] .
\end{aligned}
$$

Definition 1. The zero solution of (8) is called mean square stable if for any $\epsilon>0$, there exists a $\delta>0$ such that $\mathbb{E}|x(t)|^{2}<$ $\epsilon$ for any $t \geq 0$ provided that $\|\varphi\|_{1}^{2}<\delta$.

Definition 2. The zero solution of (8) is called asymptotically mean square stable if it is mean square stable and $\lim _{t \rightarrow \infty} \mathbb{E}|x(t)|^{2}=0$.

Definition 3. The zero solution of (8) is called stable in probability if for any $\epsilon_{1}>0$ and $\epsilon_{2}>0$, there exists $\delta>0$ such that solution $x(t)=x(t, \varphi)$ of $(8)$ satisfies

$$
\mathbb{P}\left\{x(t, \varphi)>\epsilon_{1}\right\}<\epsilon_{2}
$$

for any initial function $\varphi \in H$ such that $\mathbb{P}\{|\varphi| \leq \delta\}=1$. Here $\mathbb{P}\{\cdot\}$ is the probability of the event enclosed in braces.

Theorem 4 (see [2]). Let there exist the functional $V(t, \varphi) \in D$ such that

$$
\begin{gathered}
c_{1} \mathbb{E}|x(t)|^{2} \leq \mathbb{E} V\left(t, x_{t}\right) \leq c_{2}\left\|x_{t}\right\|_{1}^{2}, \\
\mathbb{E} L V\left(t, x_{t}\right) \leq-c_{3} \mathbb{E}|x(t)|^{2}, \quad c_{i}>0 .
\end{gathered}
$$

Then zero solution of (8) is asymptotically mean square stable.

Theorem 5 (see [2]). Let there exist the functional $V(t, \varphi) \in D$ such that

$$
c_{1}|x(t)|^{2} \leq V\left(t, x_{t}\right) \leq c_{2}\left\|x_{t}\right\|_{0}^{2}, \quad L V\left(t, x_{t}\right) \leq 0, c_{i}>0
$$

for any function $\varphi \in H$ such that $P\{|\varphi| \leq \delta\}=1$, where $\delta>0$ is sufficiently small. Then the zero solution of (8) is stable in probability.

\section{Main Results}

To prove the zero solution of system (5) is stable in probability, firstly, we will consider the linear part of system (5) and the auxiliary system without delays. Next, we will consider the auxiliary system without delays of system (5) and we will prove that the zero solution of auxiliary system (7) is asymptotic mean square stable.

Theorem 6. Suppose that

$$
\sigma_{1}^{2}<2 \mu_{1}, \quad \sigma_{2}^{2}<2 \mu_{2}, \quad\left(1+\frac{q}{p^{2}}\right) \sigma_{3}^{2}<2 \mu_{3}
$$

then zero solution of system (7) is asymptotic mean square stable.

Proof. Consider the following Lyapunov function:

$$
V_{1}=p y_{1}^{2}+y_{2}^{2}+p^{2} y_{3}^{2}+q\left(y_{1}+y_{2}+y_{3}\right)^{2},
$$

where $p, q$ are real positive constants to be chosen in the following. From the Itô formula (11), we have the following:

$$
\begin{aligned}
& L V_{1} \\
& =2 p y_{1} \dot{y}_{1}+2 p y_{2} \dot{y}_{2}+2 p^{2} y_{3} \dot{y}_{3}+2 q\left(y_{1}+y_{2}+y_{3}\right) \\
& \times\left(\dot{y}_{1}+\dot{y}_{2}+\dot{y}_{3}\right)+(p+q) \sigma_{1}^{2} y_{1}^{2}+2(1+q) \sigma_{2}^{2} y_{2}^{2} \\
& +2\left(p^{2}+q\right) \sigma_{3}^{2} y_{3}^{2} \\
& =2\left(p y_{1}+q\left(y_{1}+y_{2}+y_{3}\right)\right) \\
& \times\left(-\left(\beta I^{*}+\mu_{1}\right) y_{1}+k y_{3}\right)+2\left(y_{2}+q\left(y_{1}+y_{2}+y_{3}\right)\right) \\
& \times\left(\beta I^{*} y_{1}-\beta S^{*} y_{2}\right)+2\left(p^{2} y_{3}+q\left(y_{1}+y_{2}+y_{3}\right)\right) \\
& \times\left(\lambda y_{2}-\mu_{3} y_{3}-k y_{3}\right) \\
& +(p+q) \sigma_{1}^{2} y_{1}^{2}+(1+q) \sigma_{2}^{2} y_{2}^{2}+\left(p^{2}+q\right) \sigma_{3}^{2} y_{3}^{2} \\
& +2 q \sigma_{1} \sigma_{2} y_{1} y_{2}+2 q \sigma_{2} \sigma_{3} y_{2} y_{3} \\
& \leq+2 q \sigma_{1} \sigma_{3} y_{1} y_{3}-2 p y_{1}^{2}\left(\beta I^{*}+\mu_{1}\right)-2 q\left(y_{1}+y_{2}+y_{3}\right) \\
& \times\left(\mu_{1} y_{1}+\beta S^{*} y_{2}-\lambda y_{2}+\mu_{3} y_{3}\right)+2 \beta I^{*} y_{1} y_{2}-2 \beta S^{*} y_{2}^{2} \\
& -2 p^{2} \mu_{3} y_{3}^{2}+(p+q) \sigma_{1}^{2} y_{1}^{2} \\
& +(1+q) \sigma_{2}^{2} y_{2}^{2}+p^{2} \lambda\left(\frac{y_{2}^{2}}{p}+p y_{3}^{2}\right)+\left(p^{2}+q\right) \sigma_{3}^{2} y_{3}^{2} \\
& +2 q \sigma_{1} \sigma_{2} y_{1} y_{2}+2 q \sigma_{2} \sigma_{3} y_{2} y_{3}+2 q \sigma_{1} \sigma_{3} y_{1} y_{3} \\
& =\left[-2 p \beta I^{*}-2 p \mu_{1}-2 q \mu_{1}+(p+q) \sigma_{1}^{2}\right] \\
& +y_{2}^{2}\left[-2 q \beta S^{*}-2 \beta S^{*}+(1+q) \sigma_{2}^{2}\right] \\
& +y_{3}^{2}\left[-2 q \mu_{3}-2 p^{2} \mu_{3}+p^{2} \lambda p-2 p^{2} k+p^{2} \sigma_{3}^{2}\right] \\
& +2 y_{1} y_{2}\left[-q \mu_{1}-q \beta S^{*}+q \lambda+\beta I^{*}+q \sigma_{1} \sigma_{2}\right] \\
& +2 y_{2} y_{3}\left[-q \mu_{3}-q \beta S^{*}+q \lambda+q \sigma_{2} \sigma_{3}\right] \\
& +2 y_{1} y_{3}\left[-q \mu_{3}-q \mu_{1}+p k+q \sigma_{1} \sigma_{3}\right]+q \sigma_{3}^{2} y_{3}^{2}
\end{aligned}
$$




$$
\begin{aligned}
\leq & y_{1}^{2}(p+q)\left(\sigma_{1}^{2}-2 \mu_{1}\right) \\
& +y_{2}^{2}\left[(1+q)\left(\sigma_{2}^{2}-2 \beta S^{*}\right)+p \lambda+2 q \lambda\right] \\
& +p^{2} y_{3}^{2}\left(p \lambda-2 \mu_{3}+\sigma_{3}^{2}\right) \\
& +2 y_{1} y_{2}\left[\beta I^{*}+q\left(\sigma_{1} \sigma_{2}-\mu_{1}-\beta S^{*}+\lambda\right)\right] \\
& +2 y_{1} y_{3}\left[q\left(-\mu_{3}-\mu_{1}+\sigma_{1} \sigma_{3}\right)+p k\right] \\
& +q \sigma_{3}^{2} y_{3}^{2}+2 y_{2} y_{3}\left[q\left(-\mu_{3}-\beta S^{*}+\lambda+\sigma_{2} \sigma_{3}\right)\right] .
\end{aligned}
$$

Set

$$
q=\min \left\{\frac{p k}{\mu_{1}+\mu_{3}-\sigma_{1} \sigma_{3}}, \frac{\beta I^{*}}{\mu_{1}+\beta S^{*}-\lambda-\sigma_{1} \sigma_{2}}\right\},
$$

and $\sigma_{3}^{2}<\left(1+\left(q / p^{2}\right)\right) \sigma_{3}^{2}<2 \mu_{3}$. From the condition (15), it follows

$$
\begin{gathered}
\left|\sigma_{1} \sigma_{2}\right| \leq \frac{\sigma_{1}^{2}+\sigma_{2}^{2}}{2}<\mu_{1}+\beta S^{*}-\lambda, \\
\left|\sigma_{2} \sigma_{3}\right| \leq \frac{\sigma_{2}^{2}+\sigma_{3}^{2}}{2}<\mu_{3}+\beta S^{*}-\lambda, \\
\left|\sigma_{1} \sigma_{3}\right| \leq \frac{\sigma_{1}^{2}+\sigma_{3}^{2}}{2}<\mu_{1}+\mu_{3} .
\end{gathered}
$$

By this way, we obtain the following:

$$
\begin{aligned}
L V_{1} \leq & -y_{1}^{2}(p+q)\left(2 \mu_{1}-\sigma_{1}^{2}\right) \\
& -y_{2}^{2}\left[(1+q)\left(2 \beta S^{*}-\sigma_{2}^{2}\right)-p \lambda-2 q \lambda\right] \\
& -p^{2} y_{3}^{2}\left(2 \mu_{3}-\left(1+\frac{q}{p^{2}}\right) \sigma_{3}^{2}-p \lambda\right) .
\end{aligned}
$$

Choose constant $p$ such that

$$
\begin{aligned}
p=\frac{1}{\lambda} \min \left\{2 \mu_{3}-\left(1+\frac{q}{p^{2}}\right) \sigma_{3}^{2},\right. \\
\left.(1+q)\left(2 \beta S^{*}-\sigma_{2}^{2}-2 q \lambda\right)\right\} .
\end{aligned}
$$

From (15), it follows that there exists $c>0$ such that $L V_{1} \leq$ $-c|y|^{2}$, where $y=\left(y_{1}, y_{2}, y_{3}\right)$. From Theorem 4 , we have that the zero solution of system (7) is asymptotic mean square stable. This completes the proof.

In the second place, we will consider the linear part of system (5) and will prove that the zero solution of system (6) is asymptotic mean square stable.

Theorem 7. Suppose that

$$
\sigma_{1}^{2}<2 \mu_{1}, \quad \sigma_{2}^{2}<\frac{2 q \mu_{2}}{1+q}, \quad\left(1+\frac{q}{p^{2}}\right) \sigma_{3}^{2}<2 \mu_{3},
$$

where $q$ is defined in (18); then the zero solution of the system (6) is asymptotic mean square stable.
Proof. Consider the following Lyapunov function;

$$
V_{2}=p z_{1}^{2}+z_{2}^{2}+p^{2} z_{3}^{2}+q\left(z_{1}+z_{2}+z_{3}\right)^{2}
$$

Let $L$ be the generating operator [1] of the system (6). We compute the following:

$$
\begin{aligned}
L V_{2} & \\
= & 2\left(p z_{1}+q\left(z_{1}+z_{2}+z_{3}\right)\right) \\
& \times\left(-\left(\beta I^{*}+\mu_{1}\right) z_{1}+k z_{3}-\beta S^{*} \int_{o}^{h} f(s) z_{2}(t-s) \mathrm{d} s\right) \\
& +2\left(z_{2}+q\left(z_{1}+z_{2}+z_{3}\right)\right) \\
& \times\left(\beta I^{*} z_{1}-\beta S^{*} z_{2}+\beta S^{*} \int_{o}^{h} f(s) z_{2}(t-s) \mathrm{d} s\right) \\
& +2\left(p^{2} z_{3}+q\left(z_{1}+z_{2}+z_{3}\right)\right)\left(\lambda z_{2}-\mu_{3} z_{3}-k z_{3}\right) \\
& +(p+q) \sigma_{1}^{2} z_{1}^{2} \\
& +(1+q) \sigma_{2}^{2} z_{2}^{2}+\left(p^{2}+q\right) \sigma_{3}^{2} z_{3}^{2}+2 q \sigma_{1} \sigma_{2} z_{1} z_{2} \\
& +2 q \sigma_{2} \sigma_{3} z_{2} z_{3}+2 q \sigma_{1} \sigma_{3} z_{1} z_{3} \\
\leq & -z_{1}^{2}(p+q)\left(2 \mu_{1}-\sigma_{1}^{2}\right) \\
& -z_{2}^{2}\left[(1+q)\left(2 \beta S^{*}-\sigma_{2}^{2}\right)-p \lambda-2 q \lambda\right] \\
& -p^{2} z_{3}^{2}\left(2 \mu_{3}-\sigma_{3}^{2}-p \lambda\right)+q \sigma_{3}^{2} z_{3}^{2}+2 z_{1} z_{2} \\
& \times\left[\beta I^{*}+q\left(\sigma_{1} \sigma_{2}-\mu_{1}-\beta S^{*}+\lambda\right)\right] \\
& +2 z_{1} z_{3}\left[q\left(-\mu_{3}-\mu_{1}+\sigma_{1} \sigma_{3}\right)+p k\right] \\
& +2 z_{2} z_{3}\left[q\left(-\mu_{3}-\beta S^{*}+\lambda+\sigma_{2} \sigma_{3}\right)\right] \\
& +2 \beta S^{*}\left(z_{2}-p z_{1}\right) \int_{0}^{h} f(s) z_{2}(t-s) \mathrm{d} s .
\end{aligned}
$$

Using (18), we obtain the following:

$$
\begin{aligned}
L V_{2} \leq & -z_{1}^{2} q\left(2 \mu_{1}-\sigma_{1}^{2}\right) \\
& -z_{2}^{2}\left[(1+q)\left(2 \beta S^{*}-\sigma_{2}^{2}\right)-p \lambda-2 q \lambda\right] \\
& -p^{2} z_{3}^{2}\left(2 \mu_{3}-\sigma_{3}^{2}-p \lambda\right)+q \sigma_{3}^{2} z_{3}^{2} \\
& +p \beta S^{*}\left[z_{1}^{2}+\int_{0}^{h} f(s) z_{2}^{2}(t-s) \mathrm{d} s\right] \\
& +\beta S^{*}\left[z_{2}^{2}+\int_{0}^{h} f(s) z_{2}^{2}(t-s) \mathrm{d} s\right]
\end{aligned}
$$




$$
\begin{aligned}
= & -z_{1}^{2}\left[q\left(2 \mu_{1}-\sigma_{1}^{2}\right)-p \beta S^{*}\right] \\
& -z_{2}^{2}\left[(1+q)\left(2 \beta S^{*}-\sigma_{2}^{2}\right)-p \lambda-2 q \lambda-\beta S^{*}\right] \\
& -p^{2} z_{3}^{2}\left(2 \mu_{3}-\sigma_{3}^{2}-p \lambda\right)+q \sigma_{3}^{2} z_{3}^{2}+\beta S^{*}(1+p) \\
& \times \int_{0}^{h} f(s) z_{2}^{2}(t-s) \mathrm{d} s,
\end{aligned}
$$

and we define a Lyapunov function as follows:

$$
V_{3}=\beta S^{*}(1+p) \int_{0}^{h} f(s) \int_{t-s}^{t} z_{2}^{2}(\tau) \mathrm{d} \tau \mathrm{d} s
$$

With the application of the multidimensional Itô's formula [1], we obtain the following:

$$
L V_{3}=\beta S^{*}(1+p) z_{2}^{2}(t)-\beta S^{*}(1+p) \int_{0}^{h} f(s) z_{2}^{2}(t-s) \mathrm{d} s .
$$

Finally, we define a Lyapunov function

$$
V_{4}=V_{2}+V_{3}
$$

By (22), (25), and (27), we have the following:

$$
\begin{aligned}
L V_{4} \leq & -z_{1}^{2}\left[q\left(2 \mu_{1}-\sigma_{1}^{2}\right)-p \beta S^{*}\right] \\
& -z_{2}^{2}\left[2 q\left(\beta S^{*}-\lambda\right)-(1+q) \sigma_{2}^{2}-p\left(\lambda+\beta S^{*}\right)\right] \\
& -p^{2} z_{3}^{2}\left(2 \mu_{3}-\left(1+\frac{q}{p^{2}}\right) \sigma_{3}^{2}-p \lambda\right)
\end{aligned}
$$

where

$$
\begin{gathered}
p=\min \left\{\frac{q\left(2 \mu_{1}-\sigma_{1}^{2}\right)}{\beta S^{*}}, \frac{2 q\left(\beta S^{*}-\lambda\right)-(1+q) \sigma_{2}^{2}}{\lambda+\beta S^{*}},\right. \\
\left.\frac{2 \mu_{3}-\left(1+\left(q / p^{2}\right)\right) \sigma_{3}^{2}}{\lambda}\right\} .
\end{gathered}
$$

Therefore, there exists a $c>0$ such that $L V_{4} \leq-c|z|^{2}$, where $z=\left(z_{1}, z_{2}, z_{3}\right)$. From Theorem 4 , we have that the zero solution of system (6) is asymptotic mean square stable. This completes the proof.

Theorem 8. Suppose that the conditions of Theorem 7 hold; then the zero solution of the system (5) is stable in probability.

Proof. Consider the following Lyapunov function:

$$
\begin{aligned}
V= & p u_{1}^{2}+u_{2}^{2}+p^{2} u_{3}^{2}+q\left(u_{1}+u_{2}+u_{3}\right)^{2} \\
& +\beta S^{*}(1+p) \int_{0}^{h} f(s) \int_{t-s}^{t} u_{2}^{2}(\tau) \mathrm{d} \tau \mathrm{d} s .
\end{aligned}
$$

Then, we obtain the following:

$L V$

$$
\begin{aligned}
& =2\left(p u_{1}+q\left(u_{1}+u_{2}+u_{3}\right)\right) \\
& \times\left(-\left(\beta I^{*}+\mu_{1}\right) u_{1}+k u_{3}-\beta S^{*} \int_{0}^{h} f(s) u_{2}(t-s) \mathrm{d} s\right. \\
& \left.-\beta u_{1} \int_{0}^{h} f(s) u_{2}(t-s) \mathrm{d} s\right)+2\left(u_{2}+q\left(u_{1}+u_{2}+u_{3}\right)\right) \\
& \times\left(\beta I^{*} u_{1}-\beta S^{*} u_{2}+\beta S^{*} \int_{0}^{h} f(s) u_{2}(t-s) \mathrm{d} s\right. \\
& \left.+\beta u_{1} \int_{0}^{h} f(s) u_{2}(t-s) \mathrm{d} s\right) \\
& +2\left(p^{2} u_{3}+q\left(u_{1}+u_{2}+u_{3}\right)\right) \\
& \times\left(\lambda u_{2}-\mu_{3} u_{3}-k u_{3}\right)+(p+q) \sigma_{1}^{2} u_{1}^{2} \\
& +(1+q) \sigma_{2}^{2} u_{2}^{2}+\left(p^{2}+q\right) \sigma_{3}^{2} u_{3}^{2} \\
& +2 q \sigma_{1} \sigma_{2} u_{1} u_{2}+2 q \sigma_{2} \sigma_{3} u_{2} u_{3}+2 q \sigma_{1} \sigma_{3} u_{1} u_{3} \\
& +(1+p) \beta S^{*} u_{2}^{2}-(1+p) \beta S^{*} \int_{0}^{h} f(s) u_{2}^{2}(t-s) \mathrm{d} s \\
& \leq-u_{1}^{2}\left[q\left(2 \mu_{1}-\sigma_{1}^{2}\right)-p \beta S^{*}\right] \\
& -u_{2}^{2}\left[2 q\left(\beta S^{*}-\lambda\right)-(1+q) \sigma_{2}^{2}-p\left(\lambda+\beta S^{*}\right)\right] \\
& -p^{2} u_{3}^{2}\left(2 \mu_{3}-\sigma_{3}^{2}-p \lambda\right)+2 \beta u_{1}\left(u_{2}-p u_{1}\right) \\
& \times \int_{0}^{h} f(s) u_{2}(t-s) \mathrm{d} s .
\end{aligned}
$$

From Theorem 5 , there exists a sufficiently small $\delta>0$ such that $\mathbb{P}\left\{\left|u_{2}(s)\right|<\delta\right\}=1$. Then

$$
\begin{aligned}
2 \beta\left|u_{1}\left(u_{2}-p u_{1}\right) \int_{0}^{h} f(s) u_{2}(t-s) \mathrm{d} s\right| \\
\leq\left|2 \beta u_{1} u_{2} \int_{0}^{h} f(s) u_{2}(t-s) \mathrm{d} s\right| \\
\quad+\left|2 \beta p u_{1}^{2} \int_{0}^{h} f(s) u_{2}(t-s) \mathrm{d} s\right| \\
\leq 2 \beta u_{1} u_{2} \delta+2 \beta p u_{1}^{2} \delta=\beta \delta\left(2 u_{1} u_{2}+2 p u_{1}^{2}\right) \\
\leq \beta \delta\left(u_{1}^{2}(1+2 q)+u_{2}^{2}\right) .
\end{aligned}
$$

Therefore,

$$
L V \leq-u_{1}^{2}\left[q\left(2 \mu_{1}-\sigma_{1}^{2}\right)-p \beta S^{*}-\beta \delta(1+2 q)\right]
$$




$$
\begin{aligned}
& -u_{2}^{2}\left[2 q\left(\beta S^{*}-\lambda\right)-(1+q) \sigma_{2}^{2}-p\left(\lambda+\beta S^{*}\right)-\beta \delta\right] \\
& -p^{2} u_{3}^{2}\left(2 \mu_{3}-\sigma_{3}^{2}-p \lambda\right)
\end{aligned}
$$

Hence, for sufficiently small $\delta>0$, we obtain $L V \leq 0$. From Theorem 5, we have that the zero solution of system (5) is asymptotic mean square stable. This completes the proof.

\section{Conclusion}

In this paper, we considered the SIRS epidemic model with stochastic perturbation and distributed delays and some sufficient conditions on the stability of the zero solution are established. Further, we obtain that the white noise $\sigma_{i}(i=$ $1,2,3)$ is favorable for the stability of zero solution and the distributed time delays have no impact on the stability of zero solution. The results obtained in this paper indicate that suitable stochastic perturbation can maintain the stability of zero solution.

\section{Acknowledgment}

This work was supported by The National Natural Science Foundation of China (11261056).

\section{References}

[1] E. Beretta, V. Kolmanovskii, and L. Shaikhet, "Stability of epidemic model with time delays influenced by stochastic perturbations," Mathematics and Computers in Simulation, vol. 45, no. 3-4, pp. 269-277, 1998.

[2] I. I. Gīhman and A. V. Skorohod, The Theory of Stochastic Processes. I, Springer, Berlin, Germany, 1974.

[3] M. Carletti, "On the stability properties of a stochastic model for phage-bacteria interaction in open marine environment," Mathematical Biosciences, vol. 175, no. 2, pp. 117-131, 2002.

[4] V. B. Kolmanovskiĭ and L. E. Shaukkhet, "On one method of Lyapunov functional construction for stochastic hereditary systems," Differentsialniye uravneniya, vol. 29, no. 11, pp. 19091920, 1993 (Russian).

[5] V. Kolmanovskiı̌ and L. Shaŭkhet, "New results in stability theory for stochastic functional-differential equations (SFDEs) and their applications," in Proceedings of Dynamic Systems and Applications, pp. 167-171, Dynamic Publishers, Atlanta, Ga, USA, 1994.

[6] V. Kolmanovskii and L. Shaikhet, "General method of Lyapunov functionals construction for stability investigation of stochastic difference equations," in Dynamical Systems and Applications, vol. 4 of World Scientific Series in Applicable Analysis, pp. 397439, 1995.

[7] V. B. Kolmanovskiĭ and L. E. Shaükhet, "Stability of stochastic systems with aftereffect," Rossiǔskaya Akademiya Nauk. Avtomatika i Telemekhanika, no. 7, pp. 66-85, 1993.

[8] C. V. De-Léon and G. Gómez-Alcaraz, "Globel stability condition of delayed SIRS epidemic model for vector diseases," Revista Electrónica de Contenido Matemáico, vol. 28, no. 2, pp. 1-18, 2010.
[9] J. Zhen, Z. Ma, and M. Han, "Global stability of an SIRS epidemic model with delays," Acta Mathematica Scientia, vol. 26, no. 2, pp. 291-306, 2006. 


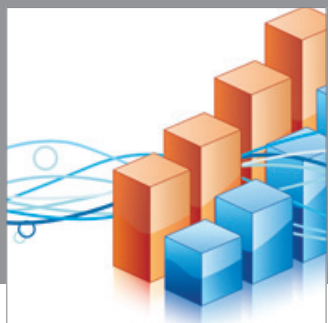

Advances in

Operations Research

mansans

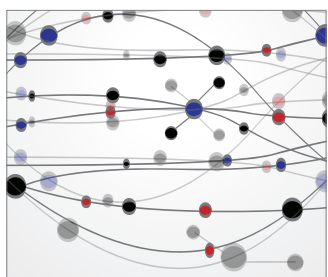

The Scientific World Journal
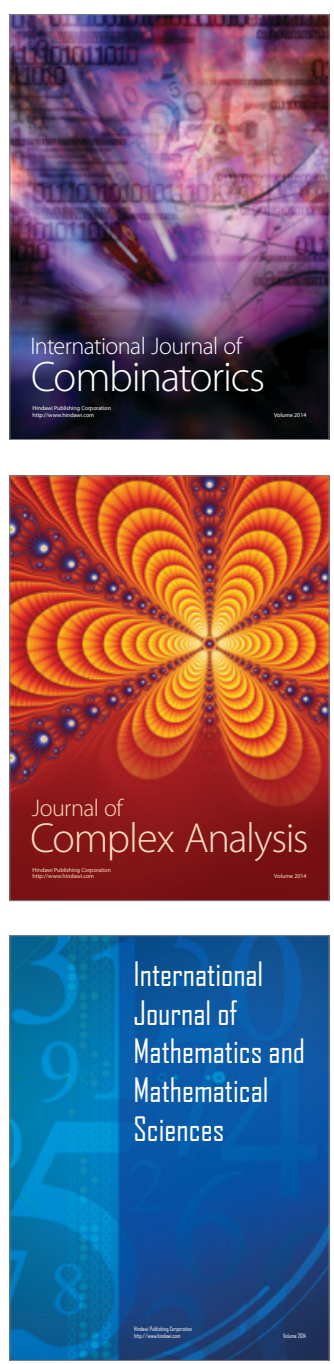
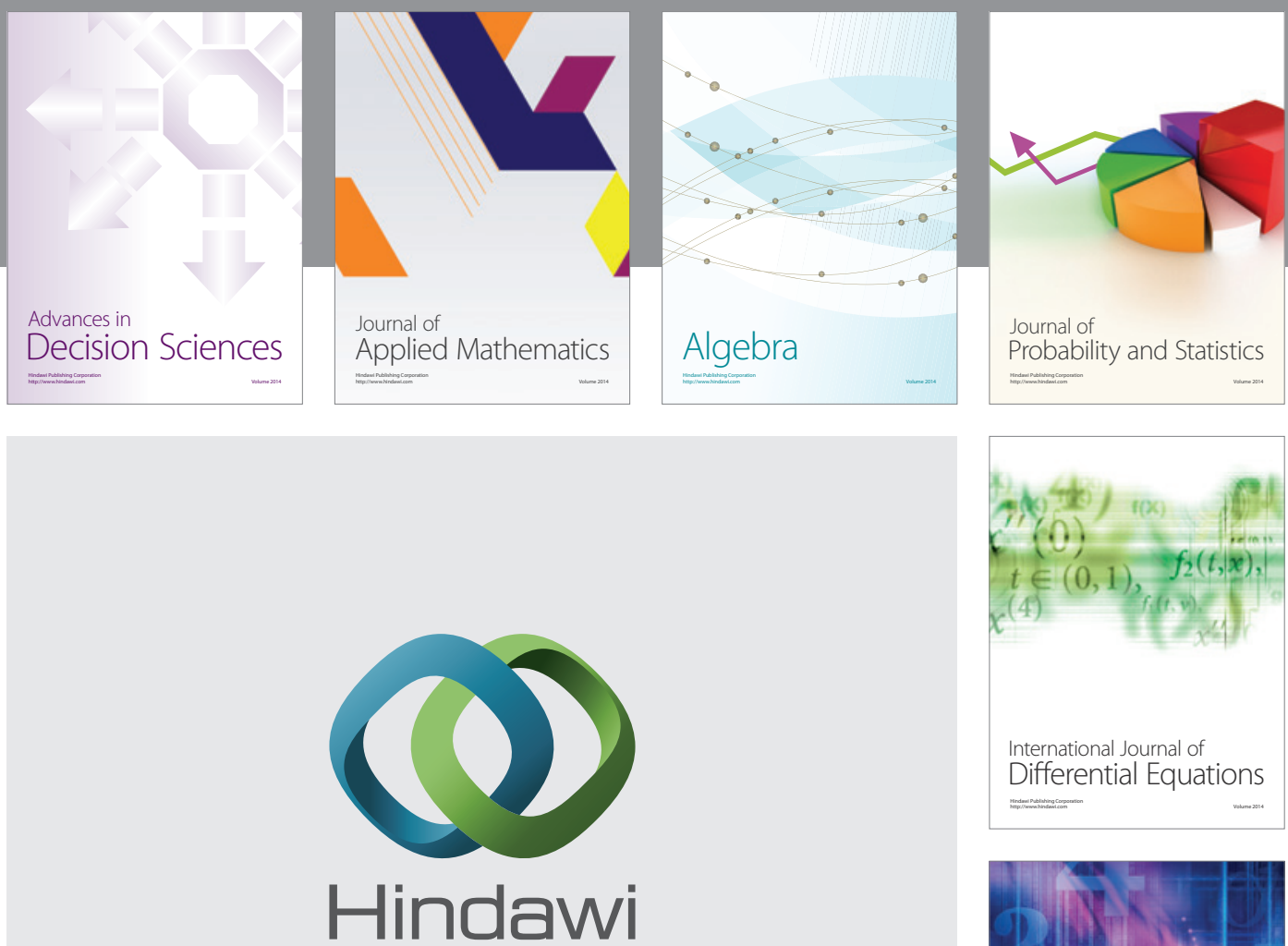

Submit your manuscripts at http://www.hindawi.com
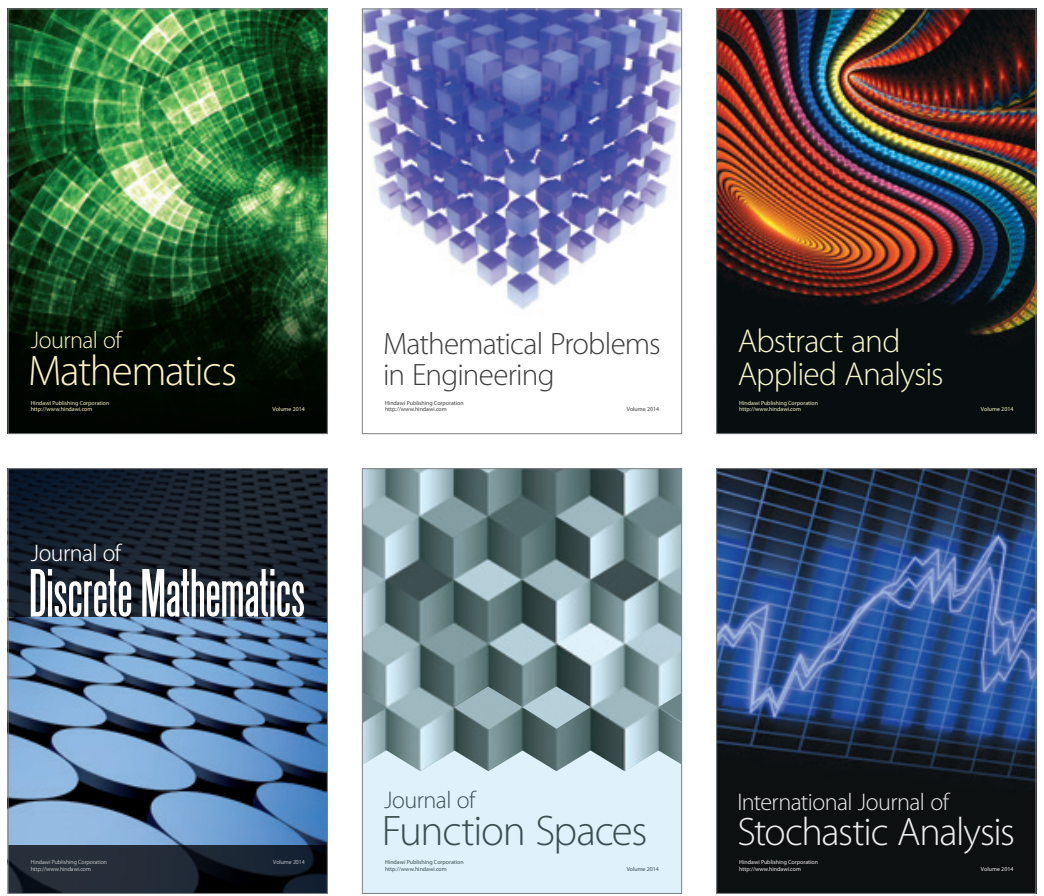

Journal of

Function Spaces

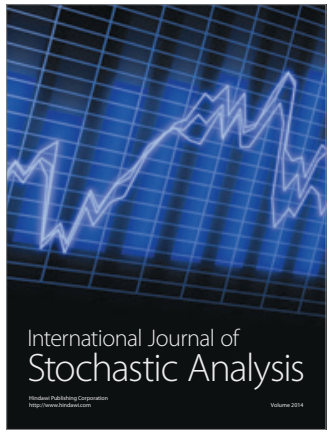

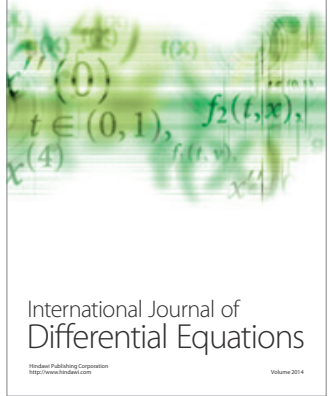
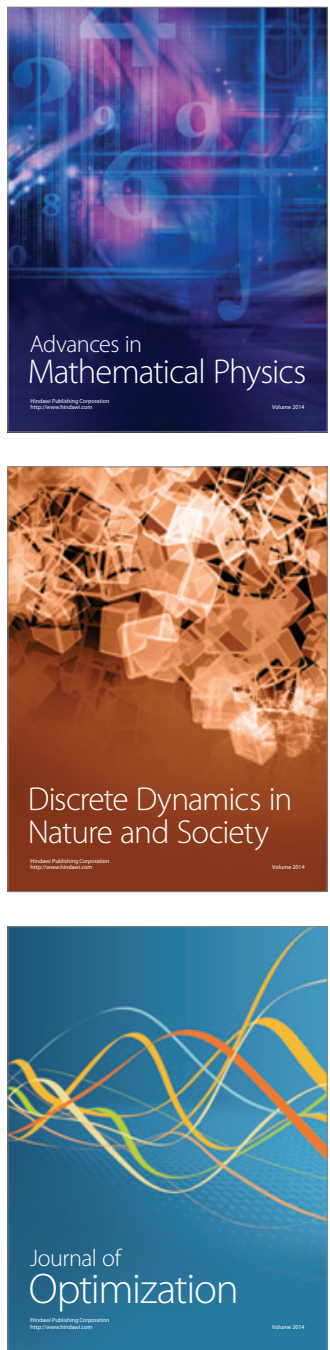